\title{
Early detection and classification of live bacteria using time-lapse coherent imaging and deep learning
}

Hongda Wang $\mathbb{E}^{1,2,3}$, Hatice Ceylan Koydemir $\mathbb{1}^{1,2,3}$, Yunzhe Qiu ${ }^{1,2,3}$, Bijie Bai ${ }^{1,2,3}$, Yibo Zhang $\mathbb{D}^{1,2,3}$, Yiyin Jin , Sabiha Tok ${ }^{1,2,3,4}$, Enis Cagatay Yilmaz', Esin Gumustekin ${ }^{5}$, Yair Rivenson ${ }^{1,2,3}$ and Aydogan Ozcan $\mathbb{B}^{1,2,3,6}$

\begin{abstract}
Early identification of pathogenic bacteria in food, water, and bodily fluids is very important and yet challenging, owing to sample complexities and large sample volumes that need to be rapidly screened. Existing screening methods based on plate counting or molecular analysis present various tradeoffs with regard to the detection time, accuracy/sensitivity, cost, and sample preparation complexity. Here, we present a computational live bacteria detection system that periodically captures coherent microscopy images of bacterial growth inside a 60-mm-diameter agar plate and analyses these time-lapsed holograms using deep neural networks for the rapid detection of bacterial growth and the classification of the corresponding species. The performance of our system was demonstrated by the rapid detection of Escherichia coli and total coliform bacteria (i.e., Klebsiella aerogenes and Klebsiella pneumoniae subsp. pneumoniae) in water samples, shortening the detection time by $>12 \mathrm{~h}$ compared to the Environmental Protection Agency (EPA)-approved methods. Using the preincubation of samples in growth media, our system achieved a limit of detection (LOD) of $\sim 1$ colony forming unit (CFU)/L in $\leq 9 \mathrm{~h}$ of total test time. This platform is highly cost-effective $(\sim 0.6 /$ test $)$ and has high-throughput with a scanning speed of $24 \mathrm{~cm}^{2} /$ min over the entire plate surface, making it highly suitable for integration with the existing methods currently used for bacteria detection on agar plates. Powered by deep learning, this automated and cost-effective live bacteria detection platform can be transformative for a wide range of applications in microbiology by significantly reducing the detection time and automating the identification of colonies without labelling or the need for an expert.
\end{abstract}

\section{Introduction}

The rapid and accurate identification of live microorganisms is of great importance for a wide range of applications ${ }^{1-8}$, including drug discovery screening assays $^{1-3}$, clinical diagnoses ${ }^{4}$, microbiome studies $^{5,6}$, and food and water safety ${ }^{7,8}$. Waterborne diseases affect more than 2 billion people worldwide ${ }^{9}$, causing a substantial

\footnotetext{
Correspondence: Aydogan Ozcan (ozcan@ucla.edu)

${ }^{1}$ Electrical and Computer Engineering Department, University of California, Los Angeles, CA 90095, USA

²Bioengineering Department, University of California, Los Angeles, CA 90095, USA

Full list of author information is available at the end of the article

These authors contributed equally: Hongda Wang, Hatice Ceylan Koydemir,

Yunzhe Qiu
}

economic burden; for example, the treatment of waterborne diseases costs more than $\$ 2$ billion annually in the United States (US) alone, with 90 million cases recorded per year ${ }^{10}$.

Among waterborne pathogen-related problems, one of the most common public health concerns is the presence of total coliform bacteria and Escherichia coli (E. coli) in drinking water, which indicates fecal contamination. Analytical methods used to detect E. coli and total coliforms are based on culturing the obtained samples on solid agar plates (e.g., the US Environmental Protection Agency (EPA) 1103.1 and EPA 1604 methods) or in liquid media (e.g., Colilert test), followed by visual recognition and counting by an expert, as described in the EPA

\section{(c) The Author(s) 2020}

(c) (i) Open Access This article is licensed under a Creative Commons Attribution 4.0 International License, which permits use, sharing, adaptation, distribution and reproduction c. in any medium or format, as long as you give appropriate credit to the original author(s) and the source, provide a link to the Creative Commons license, and indicate if changes were made. The images or other third party material in this article are included in the article's Creative Commons license, unless indicated otherwise in a credit line to the material. If material is not included in the article's Creative Commons license and your intended use is not permitted by statutory regulation or exceeds the permitted use, you will need to obtain permission directly from the copyright holder. To view a copy of this license, visit http://creativecommons.org/licenses/by/4.0/. 
guidelines ${ }^{11-13}$. While the use of liquid growth media for the detection of fecal coliform bacteria provides high sensitivity and specificity, it requires at least $18 \mathrm{~h}$ for the final read-out. The use of solid agar plates is a relatively more cost-effective method and provides flexibility for the volume of the sample to be analysed, which can vary from $100 \mathrm{~mL}$ to several litres to enhance the sensitivity. However, this traditional culture-based detection method requires the colonies to grow to a certain macroscopic size for visibility, which often takes $24-48 \mathrm{~h}$ in the case of bacterial samples. Alternatively, molecular detection methods ${ }^{14,15}$ based on, e.g., the amplification of nucleic acids, can reduce the assay time to a few hours, but they generally lack the sensitivity for detecting bacteria at very low concentrations, e.g., 1 colony forming unit (CFU) per $100-1000 \mathrm{~mL}$, and are not capable of differentiating between live and dead microorganisms ${ }^{16}$. Furthermore, there is no EPA-approved nucleic acid-based analytical method ${ }^{17}$ for detecting coliforms in water samples.

Overall, there is a strong and urgent need for an automated method that can achieve rapid and highthroughput colony detection with high sensitivity (routinely achieving, e.g., 1 CFU per $100-1000 \mathrm{~mL}$ in less than $12 \mathrm{~h}$ ) to provide a powerful alternative to the currently available EPA-approved gold-standard analytical methods that (1) are slow, take $\sim 24-48 \mathrm{~h}$ and (2) require experts to read and quantify samples. To address this important need, various other approaches ${ }^{18-20}$ have been investigated for the detection of total coliform bacteria and $E$. coli in water samples, including solid phase cytometry ${ }^{21}$, droplet-based micro-optical lens array measurements ${ }^{22}$, fluorimetry $^{23}$, luminometry ${ }^{24}$, and fluorescence microscopy $^{25}$. Despite the fact that these methods provide high sensitivity and some time savings, they cannot handle large sample sizes (e.g., $\geq 100 \mathrm{~mL}$ ) or cannot perform the automated classification of bacterial colonies.

To provide a highly sensitive and high-throughput system for the early detection and classification of live microorganisms and colony growth, we present a timelapse coherent imaging platform that uses two different deep neural networks (DNNs) for its operation. The first DNN is used to detect bacterial growth as early as possible, and the second DNN is used to classify the type of growing bacteria based on the spatiotemporal features obtained from the coherent images of an incubated agar plate (see Fig. 1). In this live bacteria detection system, which is integrated with an incubator, lens-free holographic images of the agar plate sample are captured by a monochromatic complementary metal-oxide-semiconductor (CMOS) image sensor that is mounted on a translational stage. The system rapidly scans the entire area of two separate agar plates $\left(\sim 56.52 \mathrm{~cm}^{2}\right)$ every $30 \mathrm{~min}$ and utilizes these timeresolved holographic images for the accurate detection, classification, and counting of the growing colonies as early as possible (see Fig. 2a). This unique system enables high-throughput periodic monitoring of an incubated sample by scanning a $60-\mathrm{mm}$-diameter agar plate in $87 \mathrm{~s}$ with an image resolution of $<4 \mu \mathrm{m}$; it continuously calculates differential images of the sample of interest for the early and accurate detection of bacterial growth. The spatiotemporal features of each nonstatic object on the plate are continuously analysed using deep learning to yield the count of bacterial growth and to automatically identify the type(s) of bacteria growing on the different parts of the agar plate.

We demonstrated the efficacy of this platform by performing the early detection and classification of three types of bacteria, i.e., E. coli, Klebsiella aerogenes ( $K$. aerogenes), and Klebsiella pneumoniae (K. pneumoniae), and achieved a limit of detection (LOD) of $\sim 1$ CFU/L in $\leq 9 \mathrm{~h}$ of the total test time. Moreover, we achieved detection time savings of more than $12 \mathrm{~h}$ compared to the goldstandard EPA methods ${ }^{26}$, which usually require at least $24 \mathrm{~h}$ to obtain a result. We also quantified the growth statistics of these three different species and provided a detailed growth analysis of each type of bacteria over time. Our detection and classification neural network models were built, trained and validated with $\sim 16,000$ individual colonies resulting from 71 independent experiments and were blindly tested with 965 individual colonies collected from 15 independent experiments that were never used in the training phase. In our blind testing, the trained models demonstrated an $80 \%$ detection sensitivity within $6-9 \mathrm{~h}$, a $90 \%$ detection sensitivity within $7-10 \mathrm{~h}$, and a $>95 \%$ detection sensitivity within $12 \mathrm{~h}$, while maintaining $\sim 99.2-100 \%$ precision at any time point after $7 \mathrm{~h}$, also achieving correct identification of $80 \%$ of all three the species within 7.6-12 h. In terms of the species-specific accuracy of our classification network, within $12 \mathrm{~h}$ of incubation, we achieved $\sim 97.2 \%, \sim 84.0 \%$, and $\sim 98.5 \%$ classification accuracy for $E$. coli, $K$. aerogenes, and K. pneumoniae, respectively. These results confirm the transformative potential of our platform, which not only enables the highly sensitive, rapid and cost-effective detection of live bacteria (with a cost of $\$ 0.6$ per test, including a culture plate) but also provides a powerful and versatile tool for microbiology research.

\section{Results}

We demonstrated our system by monitoring bacterial colony growth within $60-\mathrm{mm}$-diameter agar plates and quantitatively analysed the capabilities of the platform for early detection of the bacterial growth and classification of bacterial species. To demonstrate its proof-ofconcept, we aimed to automatically detect, classify, and count E. coli and coliform bacteria in water samples using our deep learning-based platform. Throughout our training and blind testing experiments, we used water 


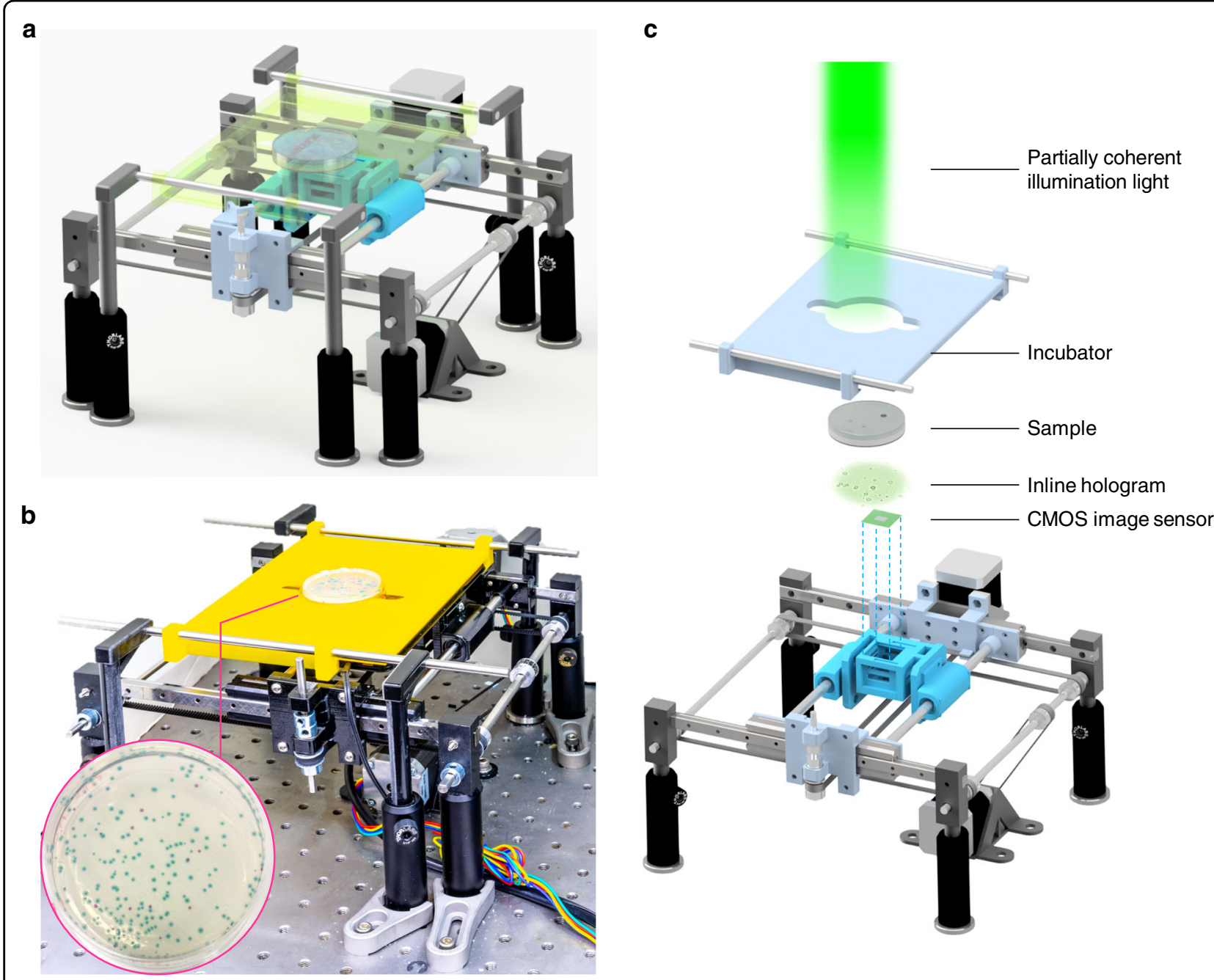

Fig. 1 High-throughput bacterial colony growth detection and classification system. a Schematic of the device. $\mathbf{b}$ Photograph of the lens-free imaging system. c Detailed illustration of various components of the system

suspensions spiked with coliform bacteria, including $E$. coli, $K$ aerogenes, and $K$. pneumoniae, and chlorinestressed E. coli. A chromogenic agar medium designed for the specific detection and counting of $E$. coli and other coliform bacteria in food and water samples was used as a culture medium for specificity (see the "Methods" section for details). This chromogenic medium results in a blue colour for $E$. coli colonies and a mauve colour for the colonies of other coliform bacteria (e.g., K. aerogenes and K. pneumoniae). In addition, the medium inhibits the growth of different bacteria (e.g., Bacillus subtilis) or yields colourless colonies in the presence of other bacteria in the sample ${ }^{27}$.

Following the sample preparation method illustrated in Fig. 2a, the sample is placed inside the lens-free imaging system with the agar surface facing the image sensor. After an initialization step, the platform automatically captures time-lapsed holographic images of two separate Petri dishes (covering a total sample area of $28.26 \times 2=56.52 \mathrm{~cm}^{2}$ ) every $30 \mathrm{~min}$ over a duration of $24 \mathrm{~h}$ starting from the incubation time; these individual holograms are digitally stitched together and rapidly reconstructed to reveal the bacterial growth patterns on the agar surface (see the "Methods" section). The reconstructed images of the sample captured at different time points are computationally processed using a differential image analysis method to automatically detect and classify bacterial growth and colonies using two different trained DNNs (see Fig. 3), which will be detailed next.

\section{Design and training of neural networks for bacterial growth detection and classification}

We designed a two-step framework for bacterial growth detection and classification. The first step selects colony candidates with differential image analysis and refines the results with a detection DNN. We designed a 


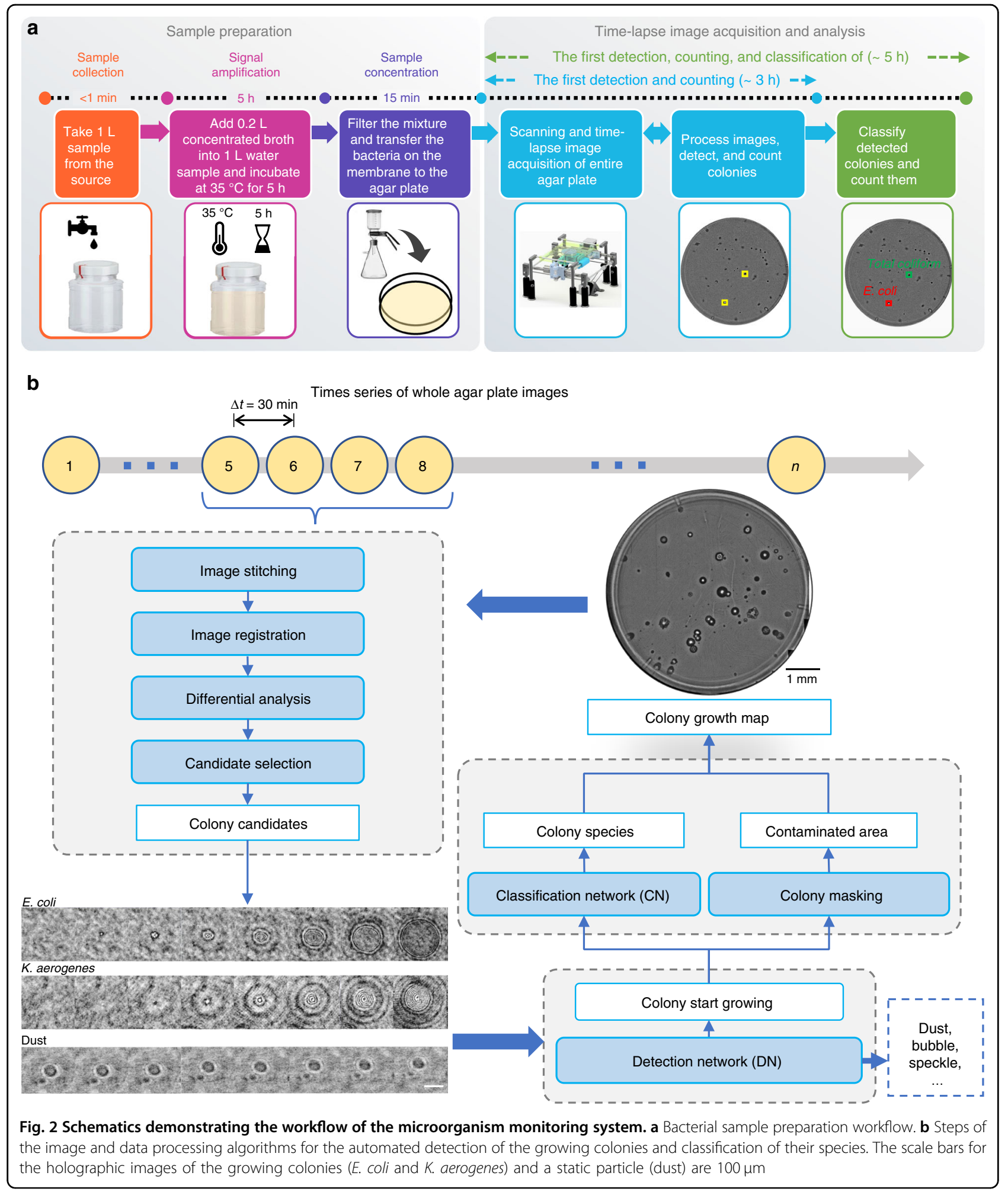

pseudo-3D (P3D) DenseNet ${ }^{28}$ architecture to process our complex-valued (i.e., phase and amplitude) timelapse image stacks (see the "Methods" section). In each time-lapse imaging experiment, we used 4 time- consecutive frames $(4 \times 0.5=2 \mathrm{~h})$ as a running window for the differential image analysis to extract individual regions of interest (ROIs) containing objects that changed their amplitude and/or phase signatures as a 

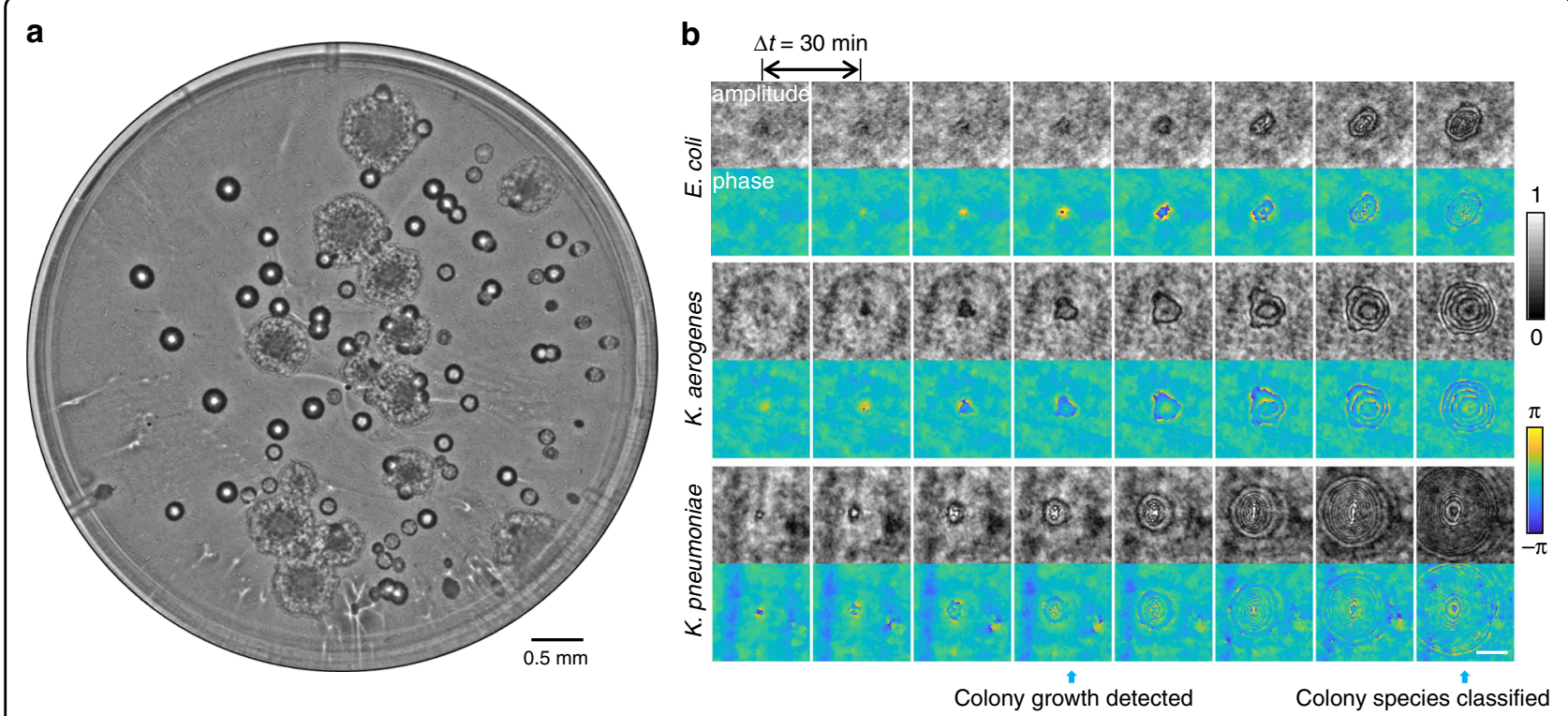

Fig. 3 Images captured using the microorganism monitoring system. a Whole agar plate image of mixed $E$. coli and $K$. aerogenes colonies after $23.5 \mathrm{~h}$ of incubation. $\mathbf{b}$ Example images (i.e., amplitude and phase) of the individual growing colonies detected by a trained deep neural network. The time points of detection and classification of growing colonies are annotated with blue arrows. The scale bar is $100 \mu \mathrm{m}$

function of time. These initially detected objects that were extracted by the differential analysis algorithm were either growing colonies or surface impurities, e.g., from spreading the sample on the agar surface, evaporation of air bubbles in the agar plate, or coherent light speckles. We then used a DNN-based detection model to eliminate the nonbacterial objects and only kept the growing colonies (i.e., the true positives), as illustrated in Fig. 2b. We used sensitivity (or true positive rate, TPR) and precision (or positive predictive value, PPV) measurements to quantify our results. Sensitivity is defined as

$$
\mathrm{TPR}=\mathrm{TP} / P
$$

where TP refers to the number of true positive predictions from our system, and $P$ refers to the total number of colonies resulting from manual plate counting after $24 \mathrm{~h}$ (i.e., the ground truth). Precision is defined as

$$
\mathrm{PPV}=\mathrm{TP} /(\mathrm{TP}+\mathrm{FP})
$$

where FP refers to the number of false positive predictions from our system.

In total, 13,712 growing colonies (E. coli, $K$. aerogenes, and $K$. pneumoniae) and 30,000 non-colony objects captured from 66 separate agar plates were used in the training phase. Another 2597 colonies and 13,078 noncolony objects from 5 independent plates were used as validation dataset to finalize our network models and achieved a TPR of $\sim 95 \%$ and a PPV of $\sim 95 \%$ once the network converged, which took $\sim 4 \mathrm{~h}$ of training time. Examples of the training loss and detection accuracy curves are shown in Supplementary Fig. S1.

The second step further classifies the species of the detected colonies with a classification DNN model following a similar network architecture. To accommodate the different growth rates of bacterial colonies, we used a longer time window in this classification neural network, containing 8 consecutive frames $(8 \times 0.5=4 \mathrm{~h})$ for each sub-ROI. Since the bacterial growth detection network uses a shorter running time window of $2 \mathrm{~h}$, there is a natural 2-h time delay between the successful detection of a growing colony and the classification of its species. The network was trained with 7919 growing colonies, which contained 3362 E. coli, $1880 \mathrm{~K}$. aerogenes, and $2677 \mathrm{~K}$. pneumoniae colonies, and it was validated with $340 \mathrm{E}$. coli, $205 \mathrm{~K}$. aerogenes, and $988 \mathrm{~K}$. pneumoniae colonies from 6 independent plates and reached a validation classification accuracy of $\sim 89 \%$ for E. coli, $\sim 95 \%$ for K. aerogenes, and $\sim 98 \%$ for K. pneumoniae when the network model converged (Supplementary Fig. S2).

After these network models were finalized through the training and validation data, we tested their generalization capabilities with an additional set of experiments that were never seen by the networks before; the results of these blind tests are detailed next. 
a

\section{K. pneumoniae (4 dishes, 339 colonies)}

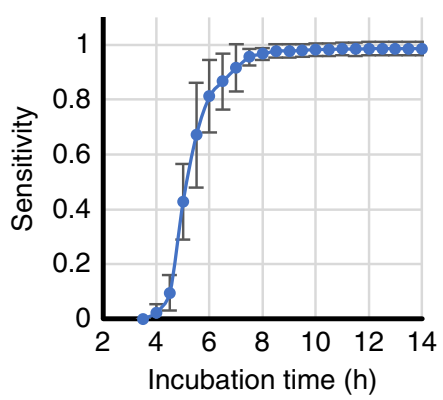

b
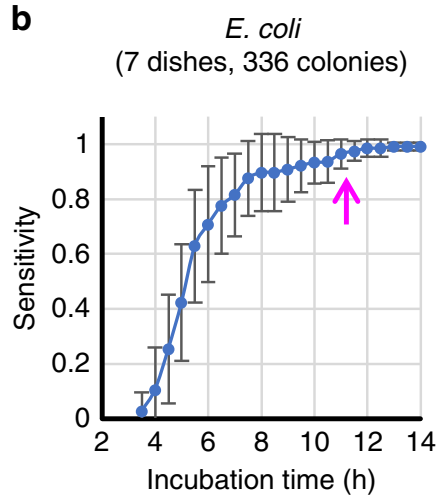

C
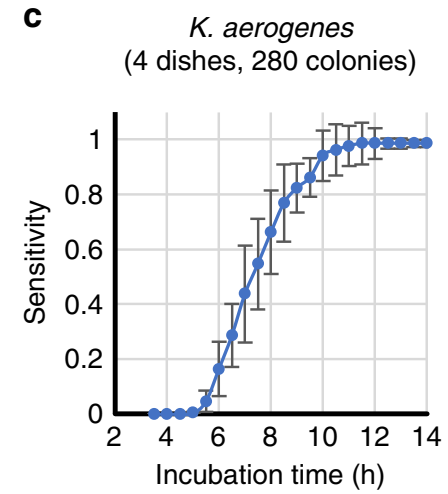

d

All experiments (15 dishes, 965 colonies)

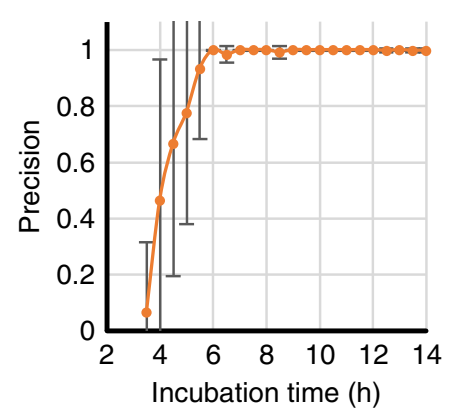

e

Normal E. coli (7 dishes, 336 colonies)

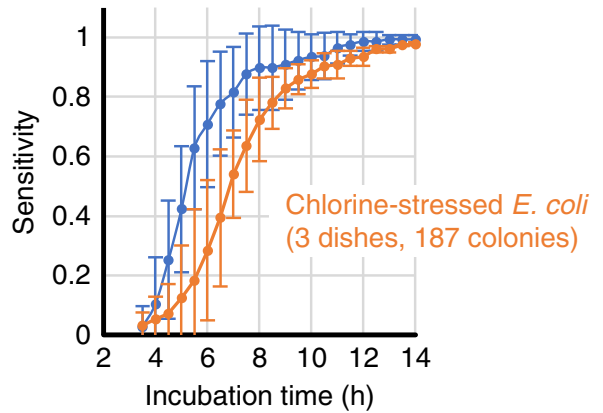

Fig. 4 Sensitivity and precision analysis. Sensitivity of growing colony detection using our trained neural network for a K. pneumoniae, $\mathbf{b} E$. coli, and $\mathbf{c} K$. aerogenes. $\mathbf{d}$ Precision of growing colony detection using our trained neural network for all three species. The pink arrow indicates the time for late "wake-up" behaviour for some of the E. coli colonies. e Characterizing the growth speed of chlorine-stressed E. coli using our system. There was an $\sim 2 \mathrm{~h}$ delay in colony formation for chlorine-stressed $E$. coli (orange curve) compared to the unstressed $E$. coli strain (blue curve). The error bars show the standard deviation values across multiple plates

\section{Blind testing results for the early detection of bacterial growth}

First, we blindly tested the performance of our system in the early detection of bacterial colonies with 965 colonies from 15 plates that were not presented during the network training or validation stages. We compared the predicted number of growing colonies on the sample within the first $14 \mathrm{~h}$ of incubation against a ground truth colony count obtained from plate counting after $24 \mathrm{~h}$ of incubation time. Each of the 3 sensitivity curves (Fig. $4 \mathrm{a}-\mathrm{c}$ ) were averaged across repeated experiments for the same species, e.g., 4 experiments for $K$. pneumoniae, 7 experiments for E. coli, and 4 experiments for K. aerogenes, so that each data point was calculated from $\sim 300$ colonies. The results demonstrated that our system was able to detect $80 \%$ of the true positive colonies within $\sim 6.0 \mathrm{~h}$ of incubation for $K$. pneumoniae, $\sim 6.8 \mathrm{~h}$ of incubation for $E$. coli, and $\sim 8.8 \mathrm{~h}$ of incubation for $K$. aerogenes. In addition, our platform further detected $90 \%$ of the true positives after $\sim 1$ additional hour of incubation and $>95 \%$ of the true positive colonies of all 3 species within $12 \mathrm{~h}$. The results also reveal that the early detection sensitivities in Fig. 4a-c are dependent on the length of the lag phase of each tested bacteria species, which demonstrates interspecies variations. For example, $K$. pneumoniae started to grow earlier and faster than $E$. coli and $K$. aerogenes, whereas $K$. aerogenes did not reach a detectable growth size until $5 \mathrm{~h}$ of incubation. Furthermore, when the tails of the sensitivity curves were examined, some of the E. coli colonies showed late "wake-up" behaviour, as highlighted by the purple arrow in Fig. 4b. Although most of the E. coli colonies were detected within $\sim 10 \mathrm{~h}$ of incubation time, some of them did not emerge until $\sim 11 \mathrm{~h}$ after the start of the incubation phase.

We also quantified the false positive rate of our platform with the PPV curve shown in Fig. 4d, which was averaged across all the experiments covering all the species, i.e., 965 colonies from 15 agar plates. The precision can be low at the beginning of the experiments (the first $4 \mathrm{~h}$ of incubation) because the number of detected true positive colonies is very small, especially for $K$. aerogenes. This result means that even a single false positive-detected colony can dramatically affect the precision calculation. 
Nevertheless, the precision quickly rises up to $~ 100 \%$ within $6 \mathrm{~h}$ of incubation and is maintained at $99.2-100 \%$ for all the tested species after $7 \mathrm{~h}$ of incubation.

We should emphasize here that the results presented in Fig. 4 represent the lower limits of the detection capabilities of our system since we calculated these sensitivities with regard to the number of true positive colonies after $24 \mathrm{~h}$ of incubation, whereas some of these colonies actually did not exist at the early stages due to delayed growth; stated differently in some cases, there were no colonies present at the early stages of the incubation period. We also note that the rising sensitivity curves in our results stand for the emergence of new bacterial colonies, in addition to the growth of colonies. Even though the sensitivity curves converge to flat lines after $12 \mathrm{~h}$, the colonies continue to grow exponentially until much later. Therefore, our system detects emerging colonies at an early stage, when they first appear, forming microscale features invisible to the naked eye.

These observations also indicate that our system can be very effective and used for high-throughput quantitative studies to better understand microorganism behaviour under different conditions, such as the evaluation of the differences in growth rates between stressed bacteria (e.g., under nutrient deprivation or chlorine treatment) and normal bacteria ${ }^{29-33}$. There are several reasons to detect and enumerate chlorine-stressed or injured coliform bacteria. First, the detection of injured $E$. coli or total coliform bacteria is directly related to the sensitivity of the detection platform ${ }^{33}$. For an effective and sensitive detection platform, false-negative results should be avoided for public health safety. Another important reason is that the detection of injured $E$. coli or low numbers of $E$. coli in water samples is correlated with Salmonella outbreaks, a foodborne pathogen causing 1.2 million illnesses and $~ 500$ deaths per year in the United States ${ }^{34}$, which forms an indirect indicator of contamination in irrigation water $^{35}$. To evaluate the capabilities of our system to detect injured bacteria, we prepared and imaged 3 agar plates containing chlorine-stressed $E$. coli (see the "Methods" section) and characterized their growth using our detection workflow, as summarized in Fig. 4e. Our results indicate that we can detect colony formation for chlorine-stressed $E$. coli on average with an $\sim 2 \mathrm{~h}$ delay compared to the regular E. coli strain.

\section{Blind testing results on the classification of growing bacteria}

In addition to providing significant detection time savings while also achieving very good sensitivity and precision for the early detection of bacterial growth, our method also provides the automated classification of the corresponding species of the detected bacteria using a trained neural network. Therefore, an additional advantage of our system is its capability to further classify the total coliform subspecies, which is not possible with traditional agar plate counting methods. For example, both $K$. pneumoniae and $K$. aerogenes colonies appear mauve in our agar plates. However, since our classification neural network not only relies on the byproducts of colorimetric reactions, it can successfully distinguish between different species based on their unique spatiotemporal growth signatures acquired by our platform at the microscale.

Figure 5 shows our blind testing results on species classification using the same experiments reported in the blinded early detection tests, containing 965 colonies of 3 different species from 15 agar plates. In these results, if a colony was not detected in the previous step (i.e., a false negative event compared to the $24 \mathrm{~h}$ reading), then it was naturally not sent to the classification neural network. We defined the recovery rate as the number of colonies correctly classified into their corresponding species using our system divided by the total number of colonies counted after $24 \mathrm{~h}$. As the classification of each individual colony is an independent event, we calculated the recovery rate for each bacterial species (reported in Fig. $5 \mathrm{a}-\mathrm{c}$ ) using all of the colonies detected in the previous step, i.e., 336, 280, and 339 colonies of E. coli, K. aerogenes, and K. pneumoniae, respectively. The shaded area in each curve represents the highest and lowest recovery rates found in all the corresponding experiments at each time point. The classification neural network correctly classified $\sim 80 \%$ of all of the colonies within $\sim 7.6, \sim 8$, and $\sim 12 \mathrm{~h}$ for $K$. pneumoniae, E. coli, and $K$. aerogenes, respectively. We once again emphasize that the results presented in Fig. 5a-c represent the lower limits of the classification capabilities of our system since ground truth is acquired after $24 \mathrm{~h}$ of incubation. In reality, at various earlier time points within the incubation period, there was no growth for certain regions of the plates, which exhibited significantly delayed growth. To further demonstrate the classification performance of our trained neural network in a manner that is decoupled from the sensitivity of the previous detection network, we report the classification confusion matrix in Fig. $5 \mathrm{~d}$ for all the colonies that were sent to the classification network for blind testing at $12 \mathrm{~h}$ after the start of the incubation. The trained network achieved classification accuracies of $\sim 97.2 \%, \sim 84.0 \%$, and $\sim 98.5 \%$ for E. coli, $K$. aerogenes, and K. pneumoniae, respectively.

\section{Limit of detection as a function of the total test time}

We further quantified the detection limit of our system and compared its performance against both Colilert ${ }^{\circledR}-18$, which is an EPA-approved method, and traditional plate counting (Supplementary Table S1, Supplementary Fig. S3). To compensate for the CFU loss during the sample transfer from the water suspension to the filter 
a

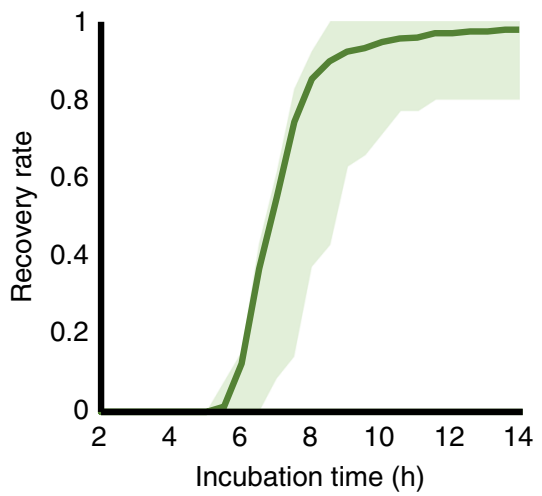

C

K. aerogenes (4 dishes, 280 colonies)

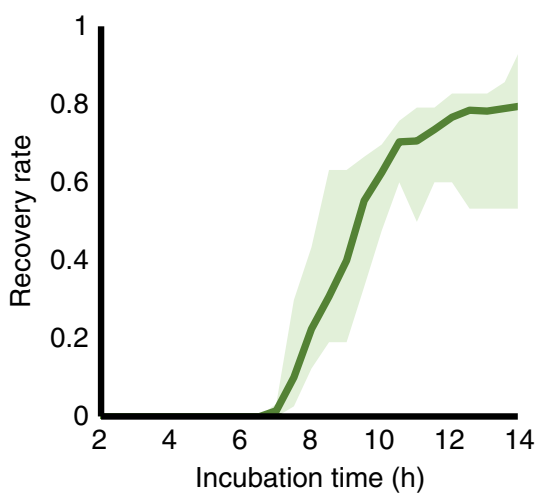

b E. coli
(7 dishes, 336 colonies)

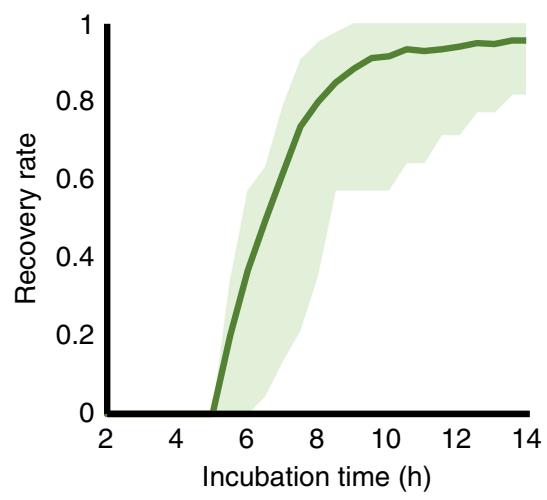

d



Fig. 5 Classification analysis. Classification performance of our trained neural network for a K. pneumoniae, $\mathbf{b}$ E. coli, and $\mathbf{c} K$. aerogenes colonies. The green shaded area in each curve represents the highest and lowest recovery rates found in all the corresponding experiments at each time point. d The blind testing confusion matrix of classifying all the colonies that were sent to our trained neural network after $12 \mathrm{~h}$ of incubation. A diagonal entry of 1.0 means a $100 \%$ classification accuracy for that species. The numbers of colonies that were tested by the classification network in $\mathbf{d}$ are 325 (E. coli), 334 (K. pneumoniae), and 256 (K. aerogenes)

membrane, we introduced a signal amplification step by preincubating the water sample under test, mixing it with a growth medium for $5 \mathrm{~h}$ at $35^{\circ} \mathrm{C}$ before the filtration step (see the "Methods" section for details). For each measurement, two agar plates were prepared and monitored at the same time for comparison, one of which was for the sample amplified with a 5 -h preincubation step before filtering, while the other was for the sample directly filtered and transferred to the agar plate (see Supplementary Fig. S3). Both plates were incubated for the same amount of time at each imaging time point to provide a fair comparison between the two. The measurements were repeated using different concentrations of $E$. coli suspensions; these concentrations were compared to the average of three replicates of the same samples prepared using the Colilert®-18 method (Supplementary Fig. S3).
As shown in Fig. 6a, our system is able to surpass the sensitivity of Colilert ${ }^{\circledR}-18$ within $\sim 8 \mathrm{~h}$ in total (including the time for signal amplification, sample concentration, and time-lapse imaging, altogether) and reach $>2$ times the sensitivity of Colilert ${ }^{\circledR}-18$ in $\sim 9 \mathrm{~h}$. We also quantified the LOD of our system by preparing and imaging 3 agar plates without bacteria, which show on average $<1 \mathrm{CFU}$ count from our setup throughout the test period from 5 to 14.5 h (Fig. 6c), revealing a detection limit of $\mu+3 \sigma=\sim 2$ CFU per test, where $\mu$ and $\sigma$ refer to the mean and standard deviation of the detected CFU count, respectively. Due to the effective signal amplification enabled by the preincubation step, even with the lowest bacterial concentration of $\sim 1 \mathrm{CFU} / \mathrm{L}$, our system was able to detect $2 \mathrm{CFU}$ at $8.5 \mathrm{~h}$ and $12 \mathrm{CFU}$ at $9 \mathrm{~h}$; in comparison, for the same contaminated water sample, Colilert ${ }^{\circledR}-18$ achieved 


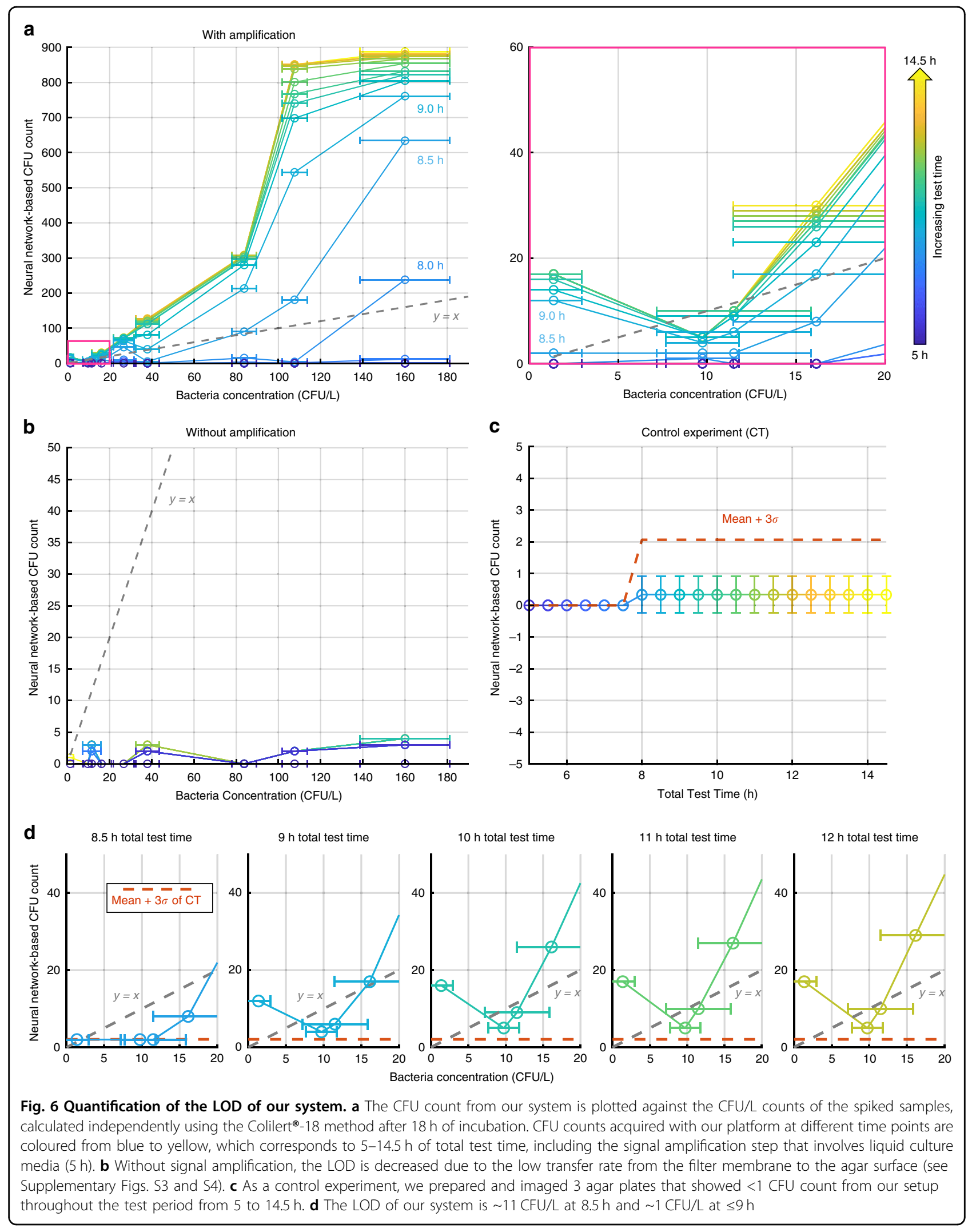


$1.4 \pm 1.6 \mathrm{CFU} / \mathrm{L}$ after $18 \mathrm{~h}$ of incubation. Furthermore, for all the concentrations we have experimented with ( 1-160 CFU/L), our system successfully detected more than 2 CFU per test in $\leq 9 \mathrm{~h}$ of test time, including all the necessary steps, i.e., the time for signal amplification, sample concentration, and time-lapse imaging; these results reveal that our system with a preincubation step achieves a detection limit of $\sim 1 \mathrm{CFU} / \mathrm{L}$ within $\leq 9 \mathrm{~h}$ of total test time.

We also observe in Fig. $6 \mathrm{~b}$ that without the signal amplification enabled by preincubation, the detection performance is negatively affected due to the low transfer rate of bacteria from the container to the agar plate (also see Supplementary Fig. S4). In general, the sensitivity and LOD of our method might be further improved by increasing the preincubation time of the water-broth mixture at the cost of an increase in the total time to achieve automated detection and classification.

\section{Discussion}

We demonstrated a new platform for the early detection and classification of bacterial colonies, which is fully compatible with the existing EPA-approved methods and can be integrated with them to considerably improve the analysis of agar plates ${ }^{36}$. The presented approach can automatically detect bacterial growth as early as $3 \mathrm{~h}$ and can detect $90 \%$ of bacterial colonies within $7-10 \mathrm{~h}$ (and $>95 \%$ within $12 \mathrm{~h}$ ), with a precision of $99.2-100 \%$. The system also correctly classifies $\sim 80 \%$ of all of the tested bacterial colonies within 7.6, 8.8, and $12 \mathrm{~h}$ for $\mathrm{K}$. pneumoniae, E. coli, and $K$. aerogenes, respectively. These results present a total time savings of more than $12 \mathrm{~h}$ compared to the gold-standard methods (e.g., Colilert test and Standard Method 9222B), which require 18-24h. The presented learning-based bacteria detection and classification framework can potentially be further advanced by training it with a larger number of sample types ${ }^{20}$, and it can also be applied to other bacteria sensing applications beyond water quality monitoring. In addition to the automated detection of live bacteria and species classification, the rich spatiotemporal information embedded in the holographic images can be used for more advanced analysis of water samples and microbiology research in general.

Another advantage of this system is its high-throughput imaging capability of agar plates. Our prototype performs a 242-tile scan within $87 \mathrm{~s}$ per agar plate, corresponding to a raw image scanning throughput of $\sim 49 \mathrm{~cm}^{2} / \mathrm{min}$. To leave sufficient data redundancy for image postprocessing, we set a relatively large overlap of $30 \%$ on each side of the acquired holographic image, which reduces the effective imaging throughput of our platform to $\sim 24 \mathrm{~cm}^{2} / \mathrm{min}$. As our system is based on lens-free holographic microscopy, it does not require mechanical axial focusing at each position and instead autofocuses onto the object plane computationally. We characterized the spatial resolution of our system by imaging a resolution test target, as shown in Supplementary Fig. S5, achieving a linewidth resolution of $\sim 3.5 \mu \mathrm{m}$, roughly equivalent to the performance of a $4 \times$ objective lens with a numerical aperture (NA) of $~ 0.1$. Compared to our system, which takes $87 \mathrm{~s}$ to scan an agar plate, a traditional lens-based bright-field microscope using a $4 \times$ objective lens would take approximately $128 \mathrm{~min}$ to scan a plate with the same diameter $(60 \mathrm{~mm})$, owing to the requirement for mechanical axial focusing (see Supplementary Table S2). In addition, the holographic imaging that is at the heart of this system provides better performance for early colony detection over brightfield imaging. Since bacteria can be considered phase objects, growth-related changes in a holographic image are enhanced compared to the bright-field images, enabling the earlier detection of bacterial growth and more sensitive measurements (see Fig. 3b).

Another important advantage of our system is the minimum requirement for optical alignment; the presented platform is tolerant towards structural changes, such as variations in the sample-to-sensor distance or the illumination angle. Our computational refocusing capability also enables the screening of thick samples, e.g., melted agar plates ${ }^{37}$. An example of a 3D sample is illustrated in Supplementary Fig. S6, where E. coli colonies are formed at different depths inside the solid culture medium with a thickness of $\sim 5 \mathrm{~mm}$. For example, the colony marked with "A" grew at $\sim 2170 \mu \mathrm{m}$ measured from the surface of the agar, whereas the colony marked with "B" was on the agar surface. Our system localizes colonies growing at different depths within a 3D culture medium using a single hologram measurement at each scanning position. However, it is a nontrivial task to image a 3D sample using a conventional lens-based microscope because of the time required for mechanical focusing and the refractive index mismatch between the culture medium and the air, which degrades the image resolution as a result of aberrations. Therefore, the corresponding brightfield microscopy images of the whole plates could only be acquired after $24 \mathrm{~h}$ of incubation.

Our platform also employs a modular design that is scalable to a larger sample size and a smaller tile-scan time interval. The monitoring field of view (FOV) of this platform is fundamentally limited by the image acquisition time and the stage moving speed. With further optimization of the hardware and control algorithms, an imaging throughput of $>50 \mathrm{~cm}^{2} / \mathrm{min}$ can be reached. Alternatively, several image sensors can be installed and connected to a single computer for high-throughput parallel imaging ${ }^{38}$. In our proof-of-concept implementation, our image processing for each time interval takes $\sim 20 \mathrm{~min}$ and fits well into our $30 \mathrm{~min}$ measurement 
period between each scan. In case a shorter time interval is desired, an image processing procedure implemented using MATLAB and Python/PyTorch programming environments can be further accelerated by programming in $\mathrm{C} / \mathrm{C}++$. With the help of graphic processing units (GPUs), one can expect $>10$-fold time savings in computation $^{39}$.

This unique platform is integrated with an incubator to keep the agar plates at a desired temperature. The incubator is a thermal glass plate that contains uniform lines of optically clear indium tin oxide electrode for heating the sample placed on top. This system is controlled with a controller, which is lightweight. Throughout the experiments, we set the temperature at the agar surface where bacteria grew at $\sim 38^{\circ} \mathrm{C}$ so that all of the tested bacterial species could grow and develop colonies. This temperature was not optimized to promote the growth of a specific species. Therefore, the adjustment of the incubation environment, temperature and humidity can potentially be used to further accelerate colony growth and help us achieve earlier detection and identification of specific bacterial colonies. Another important parameter for the growth of microorganisms is the humidity. Our system can also be integrated with a controlled humidity chamber for better control and analysis of the growth dynamics of various microorganisms ${ }^{40}$.

In summary, we presented a deep learning-based live bacteria monitoring system for the early detection of growing colonies and the classification of colony species using deep learning. We demonstrated a proof-of-concept device using 3 types of bacteria, i.e., E. coli, $K$. aerogenes, and $K$. pneumoniae, and achieved $>12 \mathrm{~h}$ time savings for both the early detection and the classification of growing species compared to the gold-standard EPA-approved methods. Achieving an LOD of $\sim 1 \mathrm{CFU} / \mathrm{L}$ in $\leq 9 \mathrm{~h}$, we believe that this versatile system will not only benefit water and food quality monitoring but also provide a powerful tool for microbiology research.

\section{Materials and methods}

\section{Sample preparation}

Safety practices

We handled all the bacterial cultures and performed all the experiments at our Biosafety Level 2 laboratory in accordance with the environmental, health, and safety rules of the University of California, Los Angeles.

\section{Studied organisms}

We used E. coli (Migula) Castellani and Chalmers (ATCC $® 25922^{\mathrm{TM}}$ ) (risk level 1), K. aerogenes Tindall et al.

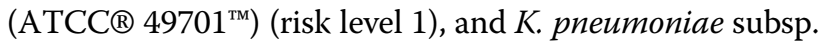
pneumoniae (Schroeter) Trevisan (ATCC®13883 ${ }^{\mathrm{TM}}$ ) (risk level 2) as our culture organisms.

\section{Preparation of the poured agar plates}

We used CHROMagar ${ }^{\mathrm{TM}}$ ECC (product no. EF322, DRG International, Inc., Springfield, NJ, USA) chromogenic substrate mixture as the solid growth medium for the detection of E. coli and total coliform colonies. CHROMagar $^{\mathrm{TM}}$ ECC $(8.2 \mathrm{~g})$ was mixed with $250 \mathrm{~mL}$ of reagent grade water (product no. 23-249-581, Fisher Scientific, Hampton, NH, USA) using a magnetic stirrer bar. The mixture was then heated to $100^{\circ} \mathrm{C}$ on a hot plate while being stirred regularly. After cooling the mixture to $\sim 50{ }^{\circ} \mathrm{C}, 10 \mathrm{~mL}$ of the mixture was dispensed into Petri dishes $(60 \mathrm{~mm} \times 15 \mathrm{~mm})$ (product no. FB0875713A, Fisher Scientific, Hampton, NH, USA). The agar plates were allowed to solidify, were sealed using parafilm (product no. 13-374-16, Fisher Scientific, Hampton, NH, USA), and were covered with aluminium foil to keep them in the dark before use. The plates were stored at $4{ }^{\circ} \mathrm{C}$ and were used within two weeks of preparation.

\section{Preparation of the melted agar plates}

CHROMagar $^{\mathrm{TM}}$ ECC $(3.28 \mathrm{~g})$ was mixed with $100 \mathrm{~mL}$ of reagent grade water using a magnetic stirrer bar, and the mixture was heated to $100^{\circ} \mathrm{C}$. After the mixture cooled to $\sim 40^{\circ} \mathrm{C}, 1 \mathrm{~mL}$ of the bacterial suspension was mixed with the agar and dispensed into Petri dishes. The plates were either incubated in a benchtop incubator (product no. 51030400, ThermoFisher Scientific, Waltham, MA, USA) or in our imaging platform (for monitoring the bacterial growth digitally).

We used tryptic soy agar to culture E. coli at $37^{\circ} \mathrm{C}$ and $K$. aerogenes at $35^{\circ} \mathrm{C}$ and nutrient agar to culture $K$. pneumoniae at $37^{\circ} \mathrm{C}$. Twenty grams of tryptic soy agar (product no. DF0369-17-6, Fisher Scientific, Hampton, $\mathrm{NH}, \mathrm{USA}$ ) or $11.5 \mathrm{~g}$ of nutrient agar (product no. DF000117-0, Fisher Scientific, Hampton, NH, USA) were suspended in $500 \mathrm{~mL}$ of reagent grade water using a magnetic stirrer bar. The mixture was boiled on a hot plate and then autoclaved at $121^{\circ} \mathrm{C}$ for $15 \mathrm{~min}$. After the mixture cooled to $\sim 50^{\circ} \mathrm{C}, 15 \mathrm{~mL}$ of the mixture was dispensed into Petri dishes $(100 \mathrm{~mm} \times 15 \mathrm{~mm})$ (product no. FB0875713, Fisher Scientific, Hampton, NH, USA), which were then sealed with parafilm and covered with aluminium foil to keep them in the dark before use. The Petri dishes were stored at $4{ }^{\circ} \mathrm{C}$ until use.

\section{Preparation of the chlorine-stressed E. coli samples}

We used E. coli grown on tryptic soy agar plates and incubated for $48 \mathrm{~h}$ at $37^{\circ} \mathrm{C}$. Disposable centrifuge tubes $(50 \mathrm{~mL})$ were used as a sample container, and the sample size was $50 \mathrm{~mL}$. Five hundred millilitres of reagent grade water was filtered for sterilization using a disposable vacuum filtration unit (product no. FB12566504, Fisher Scientific, Hampton, NH, USA). A fresh chlorine 
suspension was prepared in a $50 \mathrm{~mL}$ disposable centrifuge tube to a final concentration of $0.2 \mathrm{mg} / \mathrm{mL}$ using sodium hypochlorite (product no. 425044, Sigma Aldrich, St. Louis, MO, USA), mixed vigorously, and covered with aluminium foil ${ }^{41}$. Sodium thiosulfate $(10 \%[\mathrm{w} / \mathrm{v}])$ (product no. 217263, Sigma Aldrich, St. Louis, MO, USA) in reagent grade water was prepared, and $1 \mathrm{~mL}$ of the solution was filtered using a sterile disposable syringe and a syringe filter membrane (product no. SLGV004SL, Fisher Scientific, Hampton, NH, USA) for sterilization. Water suspensions were prepared by spiking $E$. coli into filtered water samples. Fifty microlitres of the chlorine suspension (i.e., $0.2 \mathrm{ppm}$ ) was added to the test water sample, and a timer counted the chlorine exposure time. The reaction was stopped at $10 \mathrm{~min}$ of chlorine exposure by adding $50 \mu \mathrm{L}$ sodium thiosulfate into the test water sample and vigorously mixing the solution to immediately stop the chlorination reaction. CHROMagar ${ }^{\mathrm{TM}}$ ECC plates were inoculated with $200 \mu \mathrm{L}$ of the chlorine-stressed suspension, were dried in the biosafety cabinet for at most $30 \mathrm{~min}$ and then were placed on the setup for lens-free imaging. In addition, three TSA plates and one ECC ChromoSelect Selective Agar plate (product no. 85927, Sigma Aldrich, St. Louis, MO, USA) were inoculated with $1 \mathrm{~mL}$ of the control sample (not exposed to chlorine) and $0.2 \mathrm{ppm}$ of the chlorine-stressed $E$. coli water sample and dried under a biosafety cabinet for approximately $1-2 \mathrm{~h}$ with the gentle mixing of Petri dishes at some time intervals. After drying, the plates were sealed with parafilm and incubated at $37^{\circ} \mathrm{C}$ for $24 \mathrm{~h}$. After incubation, the bacterial colonies grown on the agar plates were counted, and the E. coli concentrations of the control samples and chlorine-stressed $E$. coli samples were compared. If the achieved reduction in colony count was between 2.0 and $4.0 \mathrm{log}$, then the images of CHROMagar ${ }^{\mathrm{TM}}$ ECC plates captured using the lens-free imaging platform were used for further analysis.

\section{Preparation of the culture plates for lens-free imaging}

A bacterial suspension in a phosphate-buffered solution (PBS) (product no. 20-012-027, Fisher Scientific, Hampton, NH, USA) was prepared every day from a solid agar plate incubated for $24 \mathrm{~h}$. The concentration of the suspension was measured using a spectrophotometer (model no. ND-ONE-W, Thermo Fisher), and the suspension was then diluted in PBS to a final concentration of 1-200 CFU per $0.1 \mathrm{~mL}$. One hundred microlitres of the diluted suspension was spread on a CHROMagar ${ }^{\mathrm{TM}}$ ECC plate using an L-shaped spreader (product no. 14-665-230, Fisher Scientific, Hampton, NH, USA). The plate was covered with its lid, inverted, and incubated at $37^{\circ} \mathrm{C}$ in our optical platform (Fig. 2).

\section{Preparation of a concentrated broth}

A total of $180 \mathrm{~g}$ of tryptic soy broth (product no. R455054, Fisher Scientific, Hampton, NH, USA) was added to $1 \mathrm{~L}$ reagent grade water and heated to $100^{\circ} \mathrm{C}$ by continuously mixing using a stirrer bar. The suspension was then cooled to $50^{\circ} \mathrm{C}$ and filter sterilized using a disposable filtration unit. The broth concentrate was stored at $4{ }^{\circ} \mathrm{C}$ and used within 1 week after preparation.

\section{Preparation of samples for comparison measurements}

We evaluated the performance of our method in comparison to Colilert ${ }^{\circledR}$-18, which is an EPA-approved enzyme-based analytical method for several types of regulated water samples (e.g., drinking water, surface water, and ground water) to detect E. coli ${ }^{42}$ and for plate counting using TSA plates and ECC ChromoSelect Selective Agar plates (Supplementary Fig. S3). Two bottles of $1 \mathrm{~L}$ reagent grade water were filtered using disposable vacuum filtration units and $0.2 \mathrm{~L}$ of the concentrated broth was added into one of the $1 \mathrm{~L}$ sample bottles. The bottles were covered with aluminium foil and stored in a biosafety cabinet overnight. A glass vacuum filtration unit was used for the filtration of the $1 \mathrm{~L}$ water samples. The components of the unit were covered with aluminium foil and sterilized using an autoclave. The disposable nitrocellulose filter membranes (product no. HAWG04705, EMD Millipore, Danvers, MA, USA) used in the glass filtration unit were also sterilized using the autoclave. A bacterial suspension was prepared by spiking bacteria into $50 \mathrm{~mL}$ reagent grade water using a disposable inoculation loop from a TSA plate containing E. coli colonies. The suspension was mixed gently to obtain a uniform distribution of bacteria. Three TSA plates, 3 ECC ChromoSelect Selective Agar plates, and 4 CHROMagar $^{\mathrm{TM}} \mathrm{ECC}$ plates were removed from the refrigerator and were kept at room temperature for $30 \mathrm{~min}$.

Three bottles of $120 \mathrm{~mL}$ disposable vessels with sodium thiosulfate (product no. WV120SBST-200, IDEXX Laboratories Inc., Westbrook, ME, USA) were filled with $100 \mathrm{~mL}$ filter sterilized reagent grade water. First, $0.1 \mathrm{~mL}$ of bacterial suspension was spiked into a $1 \mathrm{~L}$ water sample, a $1.2 \mathrm{~L}$ water sample $(1 \mathrm{~L}$ water $+0.2 \mathrm{~L}$ concentrated broth), 3 bottles of $100 \mathrm{~mL}$ water samples, 3 TSA plates and 3 ECC ChromoSelect Selective Agar plates, sequentially. The timer was started immediately after adding the spike into the suspensions.

First, the suspensions on TSA plates and ECC ChromoSelect Selective Agar were spread using L-shaped disposable spreaders. Then, the water sample with broth was mixed for approximately one minute and then stored at $35^{\circ} \mathrm{C}$ for $5 \mathrm{~h}$. One Colilert@-18 reagent (product no. 98-27164-00, IDEXX Laboratories Inc., Westbrook, ME, USA) was added into each $100 \mathrm{~mL}$ bacterial suspension, and the mixture was shaken. The content of the bottle was poured into a Quanti-Tray 2000 bag (product no. 98-21675-00, IDEXX Laboratories Inc., 
Westbrook, ME, USA), and after removing bubbles in each well, the bag was sealed using Quanti-Tray Sealer (product no. 98-09462-01, IDEXX Laboratories Inc., Westbrook, ME, USA). Three bags sealed and labelled with the experimental details were incubated at $35^{\circ} \mathrm{C}$ for $18 \mathrm{~h}$. Next, $30 \mathrm{~mL}$ filtered reagent grade water was used to moisturize the membrane in the glass filtration unit, and then an E. coli-contaminated $1 \mathrm{~L}$ water sample was filtered at a pressure of $50 \mathrm{kPa}$. The bottle was rinsed using $150 \mathrm{~mL}$ of sterilized reagent grade water, and the solution was filtered on the unit (Supplementary Fig. S7). The funnel was rinsed twice using $50 \mathrm{~mL}$ of sterilized reagent grade water. After the filtration was complete, the membrane was removed and placed onto a CHROMagar ${ }^{\mathrm{TM}}$ ECC plate face down. Gentle pressure was applied on the membrane using a tweezer to remove any air bubbles between the agar and the membrane. Then, $30 \mathrm{~g}$ of weight was placed on the membrane to provide continuous pressure during the transfer of bacteria from the membrane to the agar plate (Supplementary Fig. S8). After $5 \mathrm{~min}$ of incubation, the membrane was gently peeled off from the agar surface and placed into another agar facing up. The agar containing the membrane was incubated at the benchtop incubator at $35^{\circ} \mathrm{C}$, and the agar containing the transferred bacteria was incubated at the lensfree imaging platform for time-lapse imaging. After $5 \mathrm{~h}$ of incubation, the bottle containing $1.2 \mathrm{~L}$ suspension was filtered using the same procedure as described before for filtration of a $1 \mathrm{~L}$ sample. The agar plate containing the transferred bacteria was incubated at the second sample tray of the lens-free imaging setup for time-lapse imaging, while the agar containing the membrane was incubated at the benchtop incubator.

\section{Design of the high-throughput time-resolved microorganism monitoring platform}

Our platform consists of five modules: (1) a holographic imaging system, (2) a mechanical translational system, (3) an incubation unit, (4) a control circuit, and (5) a controlling program. Each module is explained in detail below.

i. We used fibre-coupled partially coherent laser illumination (SC400-4, Fianium Ltd., Southampton, UK), with the wavelength and intensity controlled through an acousto-optic tunable filter (AOTF) device (Fianium Ltd., Southampton, UK). The device was remotely controlled with a customized program written in the $\mathrm{C}++$ programming language and ran on a controlling laptop computer (product no. EON17-SLX, Origin PC). The laser light was transmitted through the sample, i.e., the agar plate that contains the bacterial colonies, and forms an inline hologram on a CMOS image sensor (product no. acA3800-14 $\mu \mathrm{m}$, Basler AG, Ahrensburg, Germany) with a pixel size of $1.67 \mu \mathrm{m}$ and an active area of $6.4 \mathrm{~mm} \times 4.6 \mathrm{~mm}$. The CMOS image sensor was connected to the same controlling laptop computer through a universal serial bus (USB) 3.0 interface and was software-triggered within the same $\mathrm{C}++$ program. The exposure time at each scanning position was precalibrated according to the intensity distribution of the illumination light and ranged from 4 to $167 \mathrm{~ms}$. The images were saved as 8-bit bitmap files for further processing.

ii. The mechanical stage was customized with a pair of linear translation rails (Accumini 2AD10AAAHL, Thomson, Radford, VA, USA), a pair of linear bearing rods ( $8 \mathrm{~mm}$-diameter, generic), and linear bearings (LM8UU, generic), and it was aided by parts printed by a 3D printer for the joints and housing (Objet30 Pro, Stratasys, Minnesota, USA). The 2D horizontal movement was powered by two stepper motors (product no. 1124090, Kysan Electronics, San Jose, CA, USA)-one for each direction, and these motors were individually controlled using stepper motor controller chips (DRV8834, Pololu Las Vegas, NV, US). To minimize the backslash effect, the whole Petri dish was scanned following a raster scan pattern.

iii. The incubation unit was built with the top heating plate of a microscope incubator (INUBTFP-WSKMF1, Tokai Hit, Shizuoka, Japan), and it was housed by a 3D frame printed by a 3D printer. The Petri dish containing the sample was placed on the heating plate with the surface having bacteria facing downwards. The temperature was controlled by a paired controller that maintained a temperature of $47^{\circ} \mathrm{C}$ on the heating plate, resulting in a temperature of $38^{\circ} \mathrm{C}$ inside the Petri dish.

iv. The control circuit consisted of three components: a microcontroller (Arduino Micro, Arduino LLC) communicating with the computer through a USB 2.0 interface, two stepper motor driver chips (DRV8834, Pololu Las Vegas, NV, US) externally powered by a $4.2 \mathrm{~V}$ constant voltage power supply (GPS-3303, GW Instek, Montclair, CA, US), and a metal-oxide-semiconductor field-effect transistorbased digital switch (SUP75P03-07, Vishay Siliconix, Shelton, CT, United States) for controlling the CMOS sensor connection.

v. The controlling program included a graphical user interface and was developed using the $\mathrm{C}++$ programming language. External libraries including Qt (v5.9.3), AOTF (Gooch \& Housego), and Pylon (v5.0.11) were integrated.

\section{Data acquisition}

We prepared inoculated agar plates of pure bacterial colonies (see the Sample Preparation subsection under the "Methods" for details) and captured images of an entire agar plate at 30-min intervals. The illumination light was set to a 
wavelength of $532 \mathrm{~nm}$ and an intensity of $\sim 400 \mu \mathrm{W}$. To maximize the image acquisition speed, the captured images were first saved into a computer memory buffer and then were written to a hard disk by another independent thread. At the end of each experiment (i.e., after $24 \mathrm{~h}$ of incubation), the sample plate was imaged using a benchtop scanning microscope (Olympus IX83) in reflection mode, and the resulting images were automatically stitched to a full-FOV image, used for comparison. Subsequently, the plate was disposed of as solid biohazardous waste. We populated the data (i.e., timelapse lens-free images) corresponding to $\sim 6969 \mathrm{E}$. coli, $\sim 2613$ $K$. aerogenes, and $\sim 6727 \mathrm{~K}$. pneumoniae individual bacterial colonies to train and validate our models. Another 965 colonies of 3 different species from 15 independent agar plates were used to blindly test our machine learning models.

\section{Image processing and analysis}

The acquired lens-free images were processed using custom-developed image processing and deep learning algorithms. Five major image processing steps were used for the early detection and automated classification and counting of colonies. These steps are described in detail below.

\section{Image stitching to obtain the image of the entire plate area}

Following the acquisition of holographic images using the multi-threading approach, all the images within a tilescan of the whole Petri dish per wavelength were merged into a single full-FOV image. During a tile scan, the images were acquired with $\sim 30 \%$ overlap on each side of the image to calculate the relative image shifts against each other. For each image, the relative shifts against all four of the neighbouring images were calculated using a phase correlation ${ }^{43}$ method, followed by an optimization step that minimized an object function, as defined by

$$
\arg \min _{T_{V F}} \sum_{A \in V \backslash\{F\}}\left(\sum_{B \in V \backslash\{F\}}\left\|\vec{t}_{A F}-\vec{t}_{B F}-\vec{p}_{A B}\right\|^{2}\right)
$$

where $V$ is the set of all tile images, $F \in V$ is a fixed image, e.g., the image captured at the centre of the sample Petri dish, $\vec{t}_{A B}$ stands for the relative position of image $A$ with respect to image $B$, and $\vec{p}_{A B}$ is the local shift between images $A$ and $B$, calculated by the phase correlation method using the overlapping regions of the two neighbouring images, which can be formulated as

$$
\vec{p}_{A B}=(\Delta x, \Delta y)=\arg \max _{(x, y)} \mathcal{F}^{-1}\left\{\frac{\mathcal{F}\{A\} \cdot \mathcal{F}\{B\}^{*}}{\left|\mathcal{F}\{A\} \cdot \mathcal{F}\{B\}^{*}\right|}\right\}
$$

where $\mathcal{F}$ is the Fourier transform operator and $\mathcal{F}^{-1}$ is the inverse Fourier transform operator. The optimal configuration $T_{V F}=\left\{\vec{t}_{A F}: A, F \in V\right\}$ represents the relative positions of all the images with respect to the fixed image $F$, and it was used as the global position of each tile image for full-FOV image stitching. To eliminate tiles with a low signal-to-noise ratio that lead to incorrect local shift estimation values, a correlation threshold of 0.3 was applied during the optimization, meaning that if the crosscorrelation coefficient of the overlapped parts of two images was below 0.3 , the shift calculation was discarded. Once the positions of all of the tiles were obtained, they were merged into a full-FOV image of the whole Petri dish using linear blending. We defined a full-FOV image of the whole Petri dish as a "frame". All the frames were normalized so that the mean value was 50 , and they were saved as unsigned 8-bit integer (0-255) arrays.

\section{Colony candidate selection by differential analysis}

When a new frame was acquired at time $t$, it was crossregistered to the previous frame at time $t-1$ and then digitally back-propagated to the sample plane ${ }^{44,45}$ to obtain the complex light field

$$
\widetilde{B}_{t}=\mathrm{P}\left(F_{t}, \mathbf{z}\right)
$$

where $F_{t}$ is the frame at time $\mathbf{t}, \mathbf{z}$ is a surface normal vector of the sample plane obtained by digital autofocusing ${ }^{46}$ at 50 randomly spaced positions, and $P$ denotes the angular spectrum-based back-propagation operation ${ }^{44,45}$, which can be calculated by multiplying the spatial Fourier transform of the input signal and the following transfer function

$$
H_{k}\left(\nu_{x}, \nu_{y}\right)= \begin{cases}\exp \left[-j \cdot 2 \pi \frac{n \cdot z}{\lambda} \sqrt{1-\left(\frac{\lambda}{n} \nu_{x}\right)^{2}-\left(\frac{\lambda}{n} \nu_{y}\right)^{2}}\right] & \left(\nu_{x}^{2}+\nu_{y}^{2} \leq\left(\frac{n}{\lambda}\right)^{2}\right) \\ 0 & \text { otherwise }\end{cases}
$$

where $n$ is the refractive index of the medium, $\lambda$ is the illumination wavelength, and $v_{x}$ and $v_{y}$ are the spatial frequencies. This operation was followed by an inverse $2 \mathrm{D}$ Fourier transform. The resulting complex-valued reconstruction provides both the amplitude and phase images of the illuminated objects. To accommodate the large FOV of a stitched frame $(36,000 \times 36,000$ pixels $)$, digital back-propagation was performed with $2048 \times 2048$-pixel blocks, which were then merged together.

Four consecutive frames were taken, i.e., from $t-3$ to $t$, and a differential image was calculated defined by

$$
D_{t}=\operatorname{HP}\left[\operatorname{LP}\left(\frac{1}{3} \sum_{\tau=t-2}^{t}\left|\tilde{B}_{\tau}-\tilde{B}_{\tau-1}\right|\right)\right]
$$

where $D_{t}$ is the differential image at time $t, \tilde{B}_{t}$ represents the complex light field obtained by back-propagating frame $t$, and LP and HP represent low-pass and high-pass image filtering, respectively. The HP filter removes the differential signal from a slowly varying background (unwanted term), and the LP filter removes 
the high-frequency noise-introduced spatial patterns. The LP and HP filter kernels were empirically set to 5 and 100 , respectively.

Following the differential image calculation, we selected regions in the differential image with $>50$ connective pixels that are above an intensity threshold, which was empirically set to 12 . These regions are marked as colony candidates, as they give a differential signal over a period of time (covering four consecutive frames). However, some of the differential signals come from nonbacterial objects, such as a water bubble or surface movement of the agar itself. Therefore, we also used two DNNs to select the true candidates and classify their species.

\section{DNN-enabled detection of growing bacterial colonies}

Following the colony candidate selection process outlined earlier, we cropped out candidate regions of $160 \times$ 160 pixels $(\sim 267 \mu \mathrm{m} \times 267 \mu \mathrm{m})$ across the four backpropagated consecutive frames and separated the complex field into amplitude and phase channels. Therefore, each candidate region is represented by a $2 \times 4 \times 160 \times 160$ array. This four-dimensional (phase/amplitude-time- $-x-y$ ) data format differs from the traditional three-dimensional data used in image classification tasks and requires a custom-designed DNN architecture that accounts for the additional dimension of time. We designed our DNN by following the block diagram of DenseNet ${ }^{28}$ and replaced the 2D convolutional layers with P3D convolutional lay$\mathrm{ers}^{47}$, as shown in Supplementary Fig. S9. Our network was implemented in Python (v3.7.2) with the PyTorch Library (v1.0.1). The network was randomly initialized and optimized using an adaptive moment estimation (Adam) optimizer $^{48}$ with a starting learning rate of $1 \times 10^{-4}$ and a batch size of 64. To stabilize the accuracy of the network model, we also set a learning rate scheduler that decayed the learning rate by half every 20 epochs. Approximately, 16,000 growing colonies and 43,000 non-colony objects captured from 71 agar plates were used in the training and validation phases. The best network model was selected based on the best validation accuracy. Data augmentation was also applied by random $90^{\circ}$-rotations and flipping operations in the spatial dimensions. The whole training process took $\sim 5 \mathrm{~h}$ using a desktop computer with dual GPUs (GTX1080Ti, Nvidia). The decision threshold value after the softmax layer was set to 0.5 during training, i.e., positive for softmax value $>0.5$ and negative for softmax value $<0.5$, which implies equal penalty to false-positive and false-negative events. We adjusted the threshold value to 0.99 , empirically based on the training dataset before blind testing, to favour fewer false-positive events.

\section{DNN-enabled classification of the bacterial colony species}

Once the true bacterial colonies are selected, they grow for another $2 \mathrm{~h}$ to collect 8 consecutive frames, i.e.,
$4 \mathrm{~h}$, and then are sent to the second DNN as a $2 \times 8 \times$ $288 \times 288$ array for the classification of colony species. To perform the classification task, this time, the training data only contain the true colonies and their corresponding species (ground truth). The network follows a similar structure and training process as the detection model, as illustrated in Supplementary Fig. S9. The network was randomly initialized and optimized using the Adam optimizer ${ }^{48}$, with a starting learning rate of $1 \times 10^{-4}$ and a batch size of 64 . The learning rate decayed by 0.9 times every 10 epochs. To avoid overfitting to a specific plate, we discarded colony images extracted from extremely dense samples (>1000 CFU per plate). As a result, approximately 9400 growing colonies were used in the training and validation of the classification model. The whole training process took $\sim 15 \mathrm{~h}$ using a desktop computer with dual GPUs (GTX1080Ti, Nvidia).

\section{Colony counting}

The respective ground truth information on the growing colonies in each experiment was created after the sample was incubated for $>24 \mathrm{~h}$. At the boundary of the plate, the agar always forms a curved surface owing to surface tension, thereby distorting the images of the colonies. Therefore, we limited the effective imaging area to a $50 \mathrm{~mm}$-diameter circle in the centre of the agar plate. In cases where multiple colonies are closely spaced and eventually merge into one large colony (e.g., towards the end of the $24 \mathrm{~h}$ incubation period), we then used lens-free time-lapsed images to verify the true colony number when detected by our method to avoid overcounting.

\section{Calculation of the imaging throughput}

In Supplementary Table S2, we compared the imaging throughput of our system and a conventional lens-based scanning microscope in terms of the space-bandwidth product $^{49}$ using the following formula:

$$
N_{\mathrm{I}}=\alpha \cdot \mathrm{FOV} \cdot r^{2} / \delta^{2}
$$

where $N_{\mathrm{I}}$ is the effective pixel count of a frame, $\delta$ is the half-pitch resolution, $r$ is the digital sampling factor along the $x$ and $y$ directions, $\alpha=2$ represents the independent spatial information contained in the phase and amplitude images of the holographic reconstruction, and $\alpha=1$ represents the amplitude-only information contained in an image captured using the standard lens-based brightfield scanning microscope. In the lens-based microscope, we used a colour camera with a pixel size of $7.4 \mu \mathrm{m}$. Therefore, for a $4 \times$ objective lens, the image resolution is limited to $\sim 3.7 \mu \mathrm{m}$, owing to the Nyquist sampling limit. Without loss of generality, we set $r=2^{50}$. 


\section{Acknowledgements}

The authors acknowledge the funding of ARO (Contract \# W911NF-17-1-0161), Koc Group and HHMI. The authors would also like to acknowledge IDEXX Laboratories Inc. for loaning the Quanti-Tray Sealer and Drs. Janine R. Hutchison and Richard M. Ozanich from Pacific Northwest National Laboratory for sharing their assistance with the chlorination of bacterial samples.

\section{Author details}

${ }^{1}$ Electrical and Computer Engineering Department, University of California, Los Angeles, CA 90095, USA. ²Bioengineering Department, University of California, Los Angeles, CA 90095, USA. ${ }^{3}$ California NanoSystems Institute, University of California, Los Angeles, CA 90095, USA. ${ }^{4}$ Department of Biophysics, Istanbul Medical Faculty, Istanbul University, Istanbul 22000, Turkey. ${ }^{5}$ Department of Microbiology, Immunology, and Molecular Genetics, University of California, Los Angeles, CA 90095, USA. 'Department of Surgery, David Geffen School of Medicine, University of California, Los Angeles, CA 90095, USA

\section{Author contributions}

H.W., H.C.K., and Y.Q. designed and built the lens-free imaging platform and the computer algorithms. H.C.K. prepared all bacterial samples. B.B., Y.Z., Y.Y., and Y.R. contributed to the system and algorithm design. H.W., H.C.K., and Y.Q. performed the experiments on the presented system. E.C.Y. and H.C.K. captured the images of cultured plates at a benchtop microscope. H.W., Y.Q., B.B., and H.C.K. processed the experimental data. E.G. and H.C.K. prepared the chlorine injured bacterial samples. S.T. and H.C.K. prepared the agar plates for the optimization of image capture settings. H.W., H.C.K., and A.O. wrote the paper. H.C.K. and A.O. formulated the research goals and aims. A.O. supervised the research.

\section{Data availability}

The data analyzed during the study are available from the corresponding author upon reasonable request.

\section{Code availability}

The codes used to perform the study are available from the corresponding author upon reasonable request.

\section{Conflict of interest}

H.C.K, H.W., Y.R., Y.Q., and A.O. have a patent application on the invention reported in this paper.

Supplementary information is available for this paper at https://doi.org/ 10.1038/s41377-020-00358-9.

Received: 18 April 2020 Revised: 20 June 2020 Accepted: 22 June 2020 Published online: 10 July 2020

\section{References}

1. Sandgren, A. et al. Tuberculosis drug resistance mutation database. PLoS Med. 6, e1000002 (2009).

2. Arain, T. M. et al. Bioluminescence screening in vitro (Bio-Siv) assays for highvolume antimycobacterial drug discovery. Antimicrob. Agents Chemother. 40 1536-1541 (1996)

3. Jacobs, W. R. Jr. et al. Rapid assessment of drug susceptibilities of Mycobacterium tuberculosis by means of luciferase reporter phages. Science $\mathbf{2 6 0}$, 819-822 (1993).

4. Goodacre, R. et al. Rapid identification of urinary tract infection bacteria using hyperspectral whole-organism fingerprinting and artificial neural networks. Microbiology 144, 1157-1170 (1998).

5. Lagier, J. C. et al. Culturing the human microbiota and culturomics. Nat. Rev. Microbiol. 16, 540-550 (2018)

6. Fierer, N. et al. Forensic identification using skin bacterial communities. Proc. Natl Acad. Sci. USA 107, 6477-6481 (2010).

7. Koydemir, H. C. et al. Rapid imaging, detection and quantification of Giardia lamblia cysts using mobile-phone based fluorescent microscopy and machine learning. Lab a Chip 15, 1284-1293 (2015).

8. Oliver, S. P., Jayarao, B. M. \& Almeida, R. A. Foodborne pathogens in milk and the dairy farm environment: food safety and public health implications. Foodborne Pathog. Dis. 2, 115-129 (2005).
9. World Water Day. https://www.cdc.gov/healthywater/observances/wwd html?CDC_AA_refVal=https\%3A\%2F\%2Fwww.cdc.gov\%2Ffeatures\% 2Fworldwaterday\%2Findex.html (2020).

10. DeFlorio-Barker, S. et al. Estimate of incidence and cost of recreational waterborne illness on United States surface waters. Environ. Health 17, 3 (2018).

11. US Environmental Protection Agency. Method 1604: Total Coliforms and Escherichia Coli in Water by Membrane Filtration Using A Simultaneous Detection Technique (MI Medium). (Environmental Protection Agency, Office of Water, United States, 2002)

12. Current Waterborne Disease Burden Data \& Gaps | Healthy Water | CDC https://www.cdc.gov/healthywater/burden/current-data.html (2018).

13. US EPA. Analytical Methods Approved for Compliance Monitoring under the Long Term 2 Enhanced Surface Water Treatment Rule (US EPA, 2017).

14. Deshmukh, R. A. et al. Recent developments in detection and enumeration of waterborne bacteria: a retrospective minireview. MicrobiologyOpen 5, 901-922 (2016).

15. Amann, R. \& Fuchs, B. M. Single-cell identification in microbial communities by improved fluorescence in situ hybridization techniques. Nat. Rev. Microbiol. 6, 339-348 (2008).

16. Kang, D. K. et al. Rapid detection of single bacteria in unprocessed blood using Integrated Comprehensive Droplet Digital Detection. Nat. Commun. 5, 5427 (2014).

17. Title 40: Protection of Environment. Electronic Code of Federal Regulations Vol. 136.3. https://www.ecfr.gov/cgi-bin/text-idx?node=pt40.1.136 (2020).

18. Huff, K. et al. Light-scattering sensor for real-time identification of Vibrio parahaemolyticus, Vibrio vulnificus and Vibrio cholerae colonies on solid agar plate. Microb. Biotechnol. 5, 607-620 (2012).

19. Choi, J. et al. A rapid antimicrobial susceptibility test based on single-cell morphological analysis. Sci. Transl. Med. 6, 267ra174 (2014).

20. Jo, Y. et al. Holographic deep learning for rapid optical screening of anthrax spores. Sci. Adv. 3, e1700606 (2017).

21. Van Poucke, S. O. \& Nelis, H. J. A 210-min solid phase cytometry test for the enumeration of Escherichia coli in drinking water. J. Appl. Microbiol. 89 390-396 (2000)

22. Kim, M. et al. Optofluidic ultrahigh-throughput detection of fluorescent drops. Lab a Chip 15, 1417-1423 (2015)

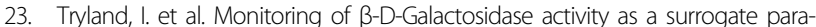
meter for rapid detection of sewage contamination in urban recreational water. Water 8, 65 (2016).

24. Van Poucke, S. O. \& Nelis, H. J. Limitations of highly sensitive enzymatic presence-absence tests for detection of waterborne coliforms and Escherichia coli. Appl. Environ. Microbiol. 63, 771-774 (1997).

25. London, R. et al. An automated system for rapid non-destructive enumeration of growing microbes. PLoS ONE 5, e8609 (2010).

26. EPA. EPA Microbiological Alternate Test Procedure (ATP) Protocol for Drinking Water, Ambient Water, Wastewater, and Sewage Sludge Monitoring Methods. (Environmental Protection Agency, Office of Water, United States, 2010).

27. CHROMagarTM ECC Product Leaflet. http://www.chromagar.com/ fichiers/1559127431LF_EXT_003_EF_V8.0.pdf?PHPSESSID= bfb3a740c98b2bf26f8ac5c4d1880fe9 (2020).

28. Huang, G. et al. Densely connected convolutional networks. 2017 IEEE Conference on Computer Vision and Pattern Recognition (CVPR) (IEEE, Honolulu, 2017).

29. Shapiro, J. A. The significances of bacterial colony patterns. BioEssays $\mathbf{1 7}$ 597-607 (1995).

30. Su, P. T. et al. Bacterial colony from two-dimensional division to threedimensional development. PLOS ONE 7, e48098 (2012).

31. Farrell, F. D. et al. Mechanical interactions in bacterial colonies and the surfing probability of beneficial mutations. J. R. Soc. Interface 14, 20170073 (2017).

32. Sheats, J. et al. Role of growth rate on the orientational alignment of Escherichia coli in a slit. R. Soc. Open Sci. 4, 170463 (2017).

33. LeChevallier, M. W. \& McFeters, G. A. Enumerating injured coliforms in drinking water. J. Am. Water Works Assoc. 77, 81-87 (1985).

34. CDC-Salmonella-Factsheet. https://www.cdc.gov/salmonella/pdf/CDCSalmonella-Factsheet.pdf (2016).

35. Liu, H. L., Whitehouse, C. A. \& Li, B. G. Presence and persistence of salmonella in water: the impact on microbial quality of water and food safety. Front. Public Health 6, 159 (2018).

36. Alternate Test Procedures in Clean Water Act Analytical Methods. https:// www.epa.gov/cwa-methods/alternate-test-procedures (2018). 
37. Sanders, E. R. Aseptic laboratory techniques: plating methods. J. Vis. Exp. https://doi.org/10.3791/3064 (2012).

38. Zhang, Y. B. et al. Motility-based label-free detection of parasites in bodily fluids using holographic speckle analysis and deep learning. Light 7, 108 (2018).

39. Isikman, S. O. et al. Lens-free optical tomographic microscope with a large imaging volume on a chip. Proc. Natl Acad. Sci. USA 108, 7296-7301 (2011).

40. Cobo, M. P. et al. Visualizing bacterial colony morphologies using time-lapse imaging chamber MOCHA. J. Bacteriol. 200, e00413-e00417 (2018).

41. Hutchison, J. R. et al. Consistent production of chlorine-stressed bacteria from non-chlorinated secondary sewage effluents for use in the U.S. Environmental Protection Agency Alternate Test Procedure protocol. J. Microbiol. Methods 163, 105651 (2019).

42. Colilert 18-IDEXX US. https:/www.idexx.com/en/water/water-productsservices/colilert-18/ (2020).

43. Preibisch, S., Saalfeld, S. \& Tomancak, P. Globally optimal stitching of tiled 3D microscopic image acquisitions. Bioinformatics 25, 1463-1465 (2009).
44. Goodman, J. W. Introduction to Fourier Optics. (Roberts and Company Publishers, Greenwoood Village, 2005).

45. Rivenson, Y. et al. Sparsity-based multi-height phase recovery in holographic microscopy. Sci. Rep. 6, 37862 (2016)

46. Zhang, Y. B. et al. Edge sparsity criterion for robust holographic autofocusing. Opt. Lett. 42, 3824-3827 (2017)

47. Qiu, Z. F., Yao, T. \& Mei, T. Learning spatio-temporal representation with pseudo-3D residual networks. 2017 IEEE International Conference on Computer Vision (ICCV) (IEEE, Venice, Italy, 2017).

48. Kingma, D. P. \& Ba, J. Adam: a method for stochastic optimization. 3rd International Conference on Learning Representations (ICLR, Ithaca, 2015)

49. Wang, H. D. et al. Computational out-of-focus imaging increases the space-bandwidth product in lens-based coherent microscopy. Optica 3, 1422-1429 (2016).

50. Greenbaum, A. et al. Increased space-bandwidth product in pixel superresolved lensfree on-chip microscopy. Sci. Rep. 3, 1717 (2013). 